\title{
Oscillation Theorems for Second-Order Half-Linear Advanced Dynamic Equations on Time Scales
}

\author{
Shuhong Tang, ${ }^{1}$ Tongxing $\mathrm{Li}^{2,3}$ and Ethiraju Thandapani ${ }^{4}$ \\ ${ }^{1}$ School of Information and Control Engineering, Weifang University, Shandong, Weifang 261061, China \\ ${ }^{2}$ School of Control Science and Engineering, Shandong University, Shandong, Jinan 250061, China \\ ${ }^{3}$ School of Mathematical Science, University of Jinan, Shandong, Jinan 250022, China \\ ${ }^{4}$ Ramanujan Institute for Advanced Study in Mathematics, University of Madras, 600005 Chennai, India
}

Correspondence should be addressed to Ethiraju Thandapani, ethandapani@yahoo.co.in

Received 7 May 2011; Revised 7 July 2011; Accepted 26 July 2011

Academic Editor: Dumitru Baleanu

Copyright @ 2011 Shuhong Tang et al. This is an open access article distributed under the Creative Commons Attribution License, which permits unrestricted use, distribution, and reproduction in any medium, provided the original work is properly cited.

This paper is concerned with the oscillatory behavior of the second-order half-linear advanced dynamic equation $\left(r(t)\left(x^{\Delta}(t)\right)^{\gamma}\right)^{\Delta}+p(t) x^{\gamma}(g(t))=0$ on an arbitrary time scale $\mathbb{T}$ with sup $\mathbb{T}=\infty$, where $g(t) \geq t$ and $\int_{t_{o}}^{\infty}\left(\Delta s /\left({ }_{r} 1 / \gamma_{(s)}\right)\right)<\infty$. Some sufficient conditions for oscillation of the studied equation are established. Our results not only improve and complement those results in the literature but also unify the oscillation of the second-order half-linear advanced differential equation and the second-order half-linear advanced difference equation. Three examples are included to illustrate the main results.

\section{Introduction}

The study of dynamic equations on time scales, which has recently received a lot of attention, was introduced by Hilger [1] in order to unify continuous and discrete analysis. Several authors have expounded on various aspects of this new theory; see the survey paper by Agarwal et al. [2] and the references cited therein. For an excellent introduction to the calculus on time scales; see Bohner and Peterson [3]. Further information on working with dynamic equations on time scales can be found in [4].

The three most popular examples of calculus on time scales are differential calculus, difference calculus, and quantum calculus, that is, when $\mathbb{T}=\mathbb{R} ; \mathbb{T}=\mathbb{N}$ and $\mathbb{T}=q^{\mathbb{N} 0}=\left\{q^{t}\right.$ : $\left.t \in \mathbb{N}_{0}\right\}$, where $q>1$. Many other interesting time scales exist, and they give rise to many applications; see [3]. Dynamic equations on a time scale have an enormous potential for applications such as in population dynamics. For example, it can model insect populations 
that are continuous while in season, die out in, say, winter, while their eggs are incubating or dormant, and then hatch in a new season, giving rise to a nonoverlapping population; see [3].

The theory of oscillations is an important branch of the applied theory of dynamic equations related to the study of oscillatory phenomena in technology and natural and social sciences. In recent years, there has been much research activity concerning the oscillation of solutions of various dynamic equations on time scales, we refer the reader to the papers [5-18] and the references therein.

We are concerned with the oscillation of the second-order half-linear advanced dynamic equation

$$
\left(r(t)\left(x^{\Delta}(t)\right)^{\gamma}\right)^{\Delta}+p(t) x^{\gamma}(g(t))=0,
$$

on a time scale $\mathbb{T}$ unbounded above, where $\gamma>0$ is the quotient of odd positive integers, $r$ and $p$ are real-valued rd-continuous positive functions defined on $\mathbb{T}, g \in C_{\mathrm{rd}}(\mathbb{T}, \mathbb{T}), g(t) \geq t$.

Since we are interested in oscillatory behavior, we assume throughout this paper that the given time scale $\mathbb{T}$ is unbounded above. We define the time scale interval of the form $\left[t_{0}, \infty\right)_{\mathbb{T}}$ by $\left[t_{0}, \infty\right)_{\mathbb{T}}:=\left[t_{0}, \infty\right) \cap \mathbb{T}$.

By a solution of (1.1), we mean a nontrivial real-valued function $x$ which has the properties $x(t) \in C_{\mathrm{rd}}^{1}\left(\left[t_{0}, \infty\right)_{\mathbb{T}}, \mathbb{R}\right), r(t)\left(x^{\Delta}(t)\right)^{\gamma} \in C_{\mathrm{rd}}^{1}\left(\left[t_{0}, \infty\right)_{\mathbb{T}}, \mathbb{R}\right)$ and satisfying $(1.1)$ for all $t \in$ $\left[t_{0}, \infty\right)_{\mathbb{T}}$. We consider only those solutions $x$ of (1.1) which satisfy $\sup \left\{|x(t)|: t \in[T, \infty)_{\mathbb{T}}\right\}>0$ for all $T \in\left[t_{0}, \infty\right)_{\mathbb{T}}$. We assume that (1.1) possesses such a solution. As usual, a solution of (1.1) is called oscillatory if it has arbitrarily large zeros on $\left[t_{0}, \infty\right)_{\mathbb{T}}$; otherwise, it is called nonoscillatory. Equation (1.1) is said to be oscillatory if all its solutions are oscillatory.

We note that if $\mathbb{T}=\mathbb{R},(1.1)$ becomes the second-order advanced differential equation

$$
\left(r(t)\left(x^{\prime}(t)\right)^{\gamma}\right)^{\prime}+p(t) x^{\gamma}(g(t))=0
$$

A special case of (1.2) is

$$
\left(r(t) x^{\prime}(t)\right)^{\prime}+p(t) x(t)=0
$$

For the oscillation of (1.3); see [19-21]. Willett [19] gave a new version of Leighton's criterion and obtained the following oscillation criteria: if

$$
\int_{t_{0}}^{\infty} \frac{1}{r(t)} \mathrm{d} t<\infty, \quad \int_{t_{0}}^{\infty} p(t)\left(\int_{t}^{\infty} \frac{\mathrm{d} s}{r(s)}\right)^{2} \mathrm{~d} t=\infty,
$$

then every solution of (1.3) is oscillatory. Later, $\mathrm{Li}$ [20] obtained that

$$
\left(t^{2} x^{\prime}(t)\right)^{\prime}+\lambda x(t)=0
$$

is oscillatory when $\lambda>1 / 4$. 
International Journal of Differential Equations

The discrete analog of (1.3) is the second-order advanced difference equation

$$
\Delta(r(n) \Delta x(n))+q(n) x(n)=0 .
$$

Budinčević [22] proved that if

$$
\sum_{n=k_{0}}^{\infty} \frac{1}{r(n)}<\infty, \quad \sum_{n=k_{0}}^{\infty} \frac{1}{r(n)} \sum_{k=k_{0}}^{n} q(k)=\infty
$$

then every solution $x$ of (1.6) is either oscillatory or else $x(n) \rightarrow 0$ as $n \rightarrow \infty$.

Regarding the oscillation of (1.1), Agarwal et al. [5], Grace et al. [7], Saker [8], and Hassan [9] studied (1.1) when $\tau(t)=t$, that is,

$$
\left(r(t)\left(x^{\Delta}(t)\right)^{\gamma}\right)^{\Delta}+p(t) x^{\gamma}(t)=0,
$$

and established some oscillation criteria for the case when

$$
\int_{t_{0}}^{\infty} \frac{\Delta t}{r^{1 / \gamma}(t)}=\infty
$$

Furthermore, the authors obtained some sufficient conditions which guarantee that every solution $x$ of (1.8) oscillates or $\lim _{t \rightarrow \infty} x(t)=0$ under the case when

$$
\int_{t_{0}}^{\infty} \frac{\Delta t}{r^{1 / \gamma}(t)}<\infty
$$

Saker [14] studied the oscillation of the dynamic equation

$$
\left(r(t) x^{\Delta}(t)\right)^{\Delta}+p(t) x(\sigma(t))=0
$$

for the cases when (1.9) and (1.10) hold with $\gamma=1$. Very recently, Hassan [15] has investigated the oscillation of (1.1) under the conditions

$$
\int_{t_{0}}^{\infty} \frac{\Delta t}{r^{1 / \gamma}(t)}=\infty \quad \text { or } \quad \int_{t_{0}}^{\infty}\left[\frac{1}{r(t)} \int_{t_{0}}^{t} p(s)\left(\int_{g(s)}^{\infty} \frac{\Delta u}{r^{1 / \gamma}(u)}\right)^{\gamma} \Delta s\right]^{1 / \gamma} \Delta t=\infty
$$

Now a problem is how to determine the oscillatory behavior of (1.1) when

$$
\int_{t_{0}}^{\infty}\left[\frac{1}{r(t)} \int_{t_{0}}^{\infty} p(s)\left(\int_{g(s)}^{\infty} \frac{\Delta u}{r^{1 / \gamma}(u)}\right)^{\gamma} \Delta s\right]^{1 / \gamma} \quad \Delta t<\infty
$$


In this paper, we will establish some new oscillation criteria for (1.1) under the case when (1.10) holds. Our results can be applied when (1.13) holds. The paper is organized as follows. In Section 2, we present some basic definitions and useful results from the theory of calculus on time scales. In Section 3, we shall establish several new oscillation criteria for (1.1).

Remark 1.1. All functional inequalities considered in this note are assumed to hold eventually, that is, they are satisfied for all $t$ large enough.

\section{Preliminary Results}

A time scale $\mathbb{T}$ is an arbitrary nonempty closed subset of the real numbers $\mathbb{R}$. Since we are interested in oscillatory behavior, we suppose that the time scale under consideration is not bounded above, that is, it is a time scale interval of the form $\left[t_{0}, \infty\right)_{\mathbb{T}}$. On any time scale, we define the forward and backward jump operators by

$$
\sigma(t):=\inf \{s \in \mathbb{T} \mid s>t\}, \quad \rho(t):=\sup \{s \in \mathbb{T} \mid s<t\}
$$

A point $t \in \mathbb{T}$ is said to be left dense if $\rho(t)=t$, right dense if $\sigma(t)=t$, left scattered if $\rho(t)<t$, and right scattered if $\sigma(t)>t$. The graininess $\mu$ of the time scale is defined by $\mu(t):=\sigma(t)-t$.

For a function $f: \mathbb{T} \rightarrow \mathbb{R}$ (the range $\mathbb{R}$ of $f$ may actually be replaced with any Banach space), the (delta) derivative is defined by

$$
f^{\Delta}(t)=\frac{f(\sigma(t))-f(t)}{\sigma(t)-t}
$$

if $f$ is continuous at $t$ and $t$ is right scattered. If $t$ is not right scattered, then the derivative is defined by

$$
f^{\Delta}(t)=\lim _{s \rightarrow t^{+}} \frac{f(\sigma(t))-f(s)}{t-s}=\lim _{s \rightarrow t^{+}} \frac{f(t)-f(s)}{t-s}
$$

provided that this limit exists.

A function $f: \mathbb{T} \rightarrow \mathbb{R}$ is said to be rd continuous if it is continuous at each right-dense point and if there exists a finite left limit in all left-dense points. The set of rd-continuous functions $f: \mathbb{T} \rightarrow \mathbb{R}$ is denoted by $C_{\mathrm{rd}}(\mathbb{T}, \mathbb{R}) . f$ is said to be differentiable if its derivative exists. The set of functions $f: \mathbb{T} \rightarrow \mathbb{R}$ that are differentiable and whose derivative is rdcontinuous function is denoted by $C_{\mathrm{rd}}^{1}(\mathbb{T}, \mathbb{R})$.

The derivative and the shift operator $\sigma$ are related by the formula

$$
f^{\sigma}(t)=f(\sigma(t))=f(t)+\mu(t) f^{\Delta}(t)
$$

Let $f$ be a real-valued function defined on an interval $[a, b]$. We say that $f$ is increasing, decreasing, nondecreasing, and nonincreasing on $[a, b]$ if $t_{1}, t_{2} \in[a, b]$ and $t_{2}>t_{1}$ imply $f\left(t_{2}\right)>f\left(t_{1}\right), f\left(t_{2}\right)<f\left(t_{1}\right), f\left(t_{2}\right) \geq f\left(t_{1}\right)$, and $f\left(t_{2}\right) \leq f\left(t_{1}\right)$, respectively. Let $f$ be a differentiable 
function on $[a, b]$. Then $f$ is increasing, decreasing, nondecreasing, and nonincreasing on $[a, b]$ if $f^{\Delta}(t)>0, f^{\Delta}(t)<0, f^{\Delta}(t) \geq 0$, and $f^{\Delta}(t) \leq 0$ for all $t \in[a, b)$, respectively.

We will make use of the following product and quotient rules for the derivative of the product $f g$ and the quotient $f / g$ (where $g(t) g(\sigma(t)) \neq 0$ ) of two differentiable functions $f$ and $g$

$$
\begin{gathered}
(f g)^{\Delta}(t)=f^{\Delta}(t) g(t)+f(\sigma(t)) g^{\Delta}(t)=f(t) g^{\Delta}(t)+f^{\Delta}(t) g(\sigma(t)), \\
\left(\frac{f}{g}\right)^{\Delta}(t)=\frac{f^{\Delta}(t) g(t)-f(t) g^{\Delta}(t)}{g(t) g(\sigma(t))} .
\end{gathered}
$$

For $a, b \in \mathbb{T}$, and a differentiable function $f$, the Cauchy integral of $f^{\Delta}$ is defined by

$$
\int_{a}^{b} f^{\Delta}(t) \Delta t=f(b)-f(a)
$$

The integration by parts formula reads

$$
\int_{a}^{b} f^{\Delta}(t) g(t) \Delta t=f(b) g(b)-f(a) g(a)-\int_{a}^{b} f^{\sigma}(t) g^{\Delta}(t) \Delta t
$$

and infinite integrals are defined as

$$
\int_{a}^{\infty} f(s) \Delta s=\lim _{t \rightarrow \infty} \int_{a}^{t} f(s) \Delta s
$$

\section{Main Results}

In this section, by employing the Riccati transformation technique, we establish several oscillation criteria for (1.1). To prove the main theorems, we will use the following formula:

$$
\left(x^{\gamma}(t)\right)^{\Delta}=\gamma \int_{0}^{1}\left[h x^{\sigma}(t)+(1-h) x(t)\right]^{\gamma-1} \mathrm{~d} h x^{\Delta}(t)
$$

which is a simple consequence of Keller's chain rule [3, Theorem 1.90].

Set

$$
d_{+}(t):=\max \{0, d(t)\}, \quad R(t):=\int_{t}^{\infty} \frac{\Delta s}{r^{1 / \gamma}(s)}
$$

and we assume that there exists a positive real-valued $\Delta$-differentiable function $m$ such that

$$
\frac{m(t)}{r^{1 / \gamma}(t) R(t)}+m^{\Delta}(t) \leq 0
$$

In order to prove the main results conveniently, we give the following known result. 
Theorem A (see [12, Theorem 2.1]). Assume that (1.9) holds. Further, assume that there exists a positive real-valued $\Delta$-differentiable function $\delta$ such that

$$
\limsup _{t \rightarrow \infty} \int_{T}^{t}\left[\delta(s) p(s)-\frac{r(s)\left(\left(\delta^{\Delta}(s)\right)_{+}\right)^{\gamma+1}}{(\gamma+1)^{\gamma+1} \delta^{\gamma}(s)}\right] \Delta s=\infty
$$

holds for all sufficiently large T. Then every solution of (1.1) is oscillatory.

Remark 3.1. From the proof of [12, Theorem 2.1], if we let $x$ be an eventually positive solution of (1.1), then $x^{\Delta}(t)>0$ due to condition (1.9). Hence, we can get a contradiction to (3.4) when $x^{\Delta}(t)>0$ occurs.

Theorem 3.2. Assume that (1.10) holds, $g(t) \geq \sigma(t)$ and $\gamma \leq 1$. Furthermore, assume that there exists a positive real-valued $\Delta$-differentiable function $\delta$ such that (3.4) holds for all sufficiently large T. If

$$
\limsup _{t \rightarrow \infty} \int_{T}^{t}\left[p(s)\left(\frac{m(g(s))}{m(\sigma(s))}\right)^{\gamma} R^{\gamma \sigma}(s)-\left(\frac{\gamma}{\gamma+1}\right)^{\gamma+1} \frac{1}{R^{\sigma}(s) r^{1 / \gamma}(s)}\right] \Delta s=\infty,
$$

then (1.1) is oscillatory.

Proof. Let $x$ be a nonoscillatory solution of (1.1). Without loss of generality, we assume that $x(t)>0$ and $x(g(t))>0$ for $t \in\left[t_{0}, \infty\right)_{\mathbb{T}}$. In view of $(1.1)$, we obtain

$$
\left(r(t)\left(x^{\Delta}(t)\right)^{\gamma}\right)^{\Delta}=-p(t) x^{\gamma}(g(t))<0, \quad t \in\left[t_{0}, \infty\right)_{\mathbb{T}} .
$$

Hence, $r(t)\left(x^{\Delta}(t)\right)^{\gamma}$ is an eventually strictly decreasing function, and there exists a $t_{1} \in$ $\left[t_{0}, \infty\right)_{\mathbb{T}}$ such that $x^{\Delta}(t)>0, t \in\left[t_{1}, \infty\right)_{\mathbb{T}}$ or $x^{\Delta}(t)<0, t \in\left[t_{1}, \infty\right)_{\mathbb{T}}$.

Case 1. Assume that $x^{\Delta}(t)>0, t \in\left[t_{1}, \infty\right)_{\mathbb{T}}$. From Theorem A, we can obtain a contradiction to $(3.4)$.

Case 2. Assume that $x^{\Delta}(t)<0, t \in\left[t_{1}, \infty\right)_{\mathbb{T}}$. Define the function $\omega$ by

$$
\omega(t)=\frac{r(t)\left(x^{\Delta}(t)\right)^{\gamma}}{x^{\gamma}(t)}
$$

Then, $\omega(t)<0$ for $t \in\left[t_{1}, \infty\right)_{\mathbb{T}}$. By (3.6), we get

$$
x^{\Delta}(s) \leq \frac{r^{1 / \gamma}(t)}{r^{1 / \gamma}(s)} x^{\Delta}(t), \quad s \in[t, \infty)_{\mathbb{T}} .
$$


Integrating it from $t$ to $l$, we have

$$
x(l) \leq x(t)+r^{1 / \gamma}(t) x^{\Delta}(t) \int_{t}^{l} \frac{\Delta s}{r^{1 / \gamma}(s)}, \quad l \in[t, \infty)_{\mathbb{T}} .
$$

Taking $l \rightarrow \infty$ in the last inequality, we get

$$
x(t)+r^{1 / \gamma}(t) x^{\Delta}(t) R(t) \geq 0, \quad t \in\left[t_{1}, \infty\right)_{\mathbb{T}} .
$$

Thus, we obtain

$$
r^{1 / \gamma}(t) R(t) \frac{x^{\Delta}(t)}{x(t)} \geq-1
$$

By (3.7) and (3.11), we have

$$
-1 \leq R^{\gamma}(t) \omega(t) \leq 0 .
$$

On the other hand, it follows from (3.11) that

$$
\frac{x^{\Delta}(t)}{x(t)} \geq-\frac{1}{r^{1 / \gamma}(t) R(t)} .
$$

Then, we have

$$
\left(\frac{x(t)}{m(t)}\right)^{\Delta}=\frac{x^{\Delta}(t) m(t)-x(t) m^{\Delta}(t)}{m(t) m^{\sigma}(t)} \geq-\frac{x(t)}{m(t) m^{\sigma}(t)}\left[\frac{m(t)}{r^{1 / \gamma}(t) R(t)}+m^{\Delta}(t)\right] \geq 0
$$

Thus, $x(t) / m(t)$ is nondecreasing. Hence we obtain

$$
\frac{x(g(t))}{x(\sigma(t))} \geq \frac{m(g(t))}{m(\sigma(t))}, \quad \text { since } g(t) \geq \sigma(t)
$$

$\Delta$-differentiating (3.7) and using (3.6), we obtain

$$
\omega^{\Delta}(t) \leq-p(t)\left(\frac{m(g(t))}{m(\sigma(t))}\right)^{\gamma}-\frac{r(t)\left(x^{\Delta}(t)\right)^{\gamma}\left(x^{\gamma}(t)\right)^{\Delta}}{x^{\gamma}(t) x^{\gamma}(\sigma(t))} .
$$

In view of Keller's chain rule [3, Theorem 1.90], we see that

$$
\left(x^{\gamma}(t)\right)^{\Delta} \leq \gamma x^{\gamma-1}(t) x^{\Delta}(t), \text { since } \gamma \leq 1 \text {. }
$$


Thus, (3.16) yields

$$
\omega^{\Delta}(t) \leq-p(t)\left(\frac{m(g(t))}{m(\sigma(t))}\right)^{\gamma}-\gamma \frac{r(t)\left(x^{\Delta}(t)\right)^{\gamma+1}}{x(t) x^{\gamma}(\sigma(t))} .
$$

On the other hand, from $x^{\Delta}(t)<0$, we have $x(t) \geq x^{\sigma}(t)$ and

$$
-\gamma \frac{r(t)\left(x^{\Delta}(t)\right)^{\gamma+1}}{x(t) x^{\gamma}(\sigma(t))} \leq-\gamma\left(\frac{1}{r(t)}\right)^{1 / \gamma} \omega^{(\gamma+1) / \gamma}(t)
$$

Hence by (3.18), we have

$$
\omega^{\Delta}(t)+p(t)\left(\frac{m(g(t))}{m(\sigma(t))}\right)^{\gamma}+\gamma r^{-1 / \gamma}(t) \omega^{(\gamma+1) / \gamma}(t) \leq 0, \quad t \in\left[t_{1}, \infty\right)_{\mathbb{T}} .
$$

Multiplying (3.20) by $R^{\gamma \sigma}(t)$, we obtain

$$
R^{\gamma \sigma}(t) \omega^{\Delta}(t)+p(t)\left(\frac{m(g(t))}{m(\sigma(t))}\right)^{\gamma} R^{\gamma \sigma}(t)+\gamma R^{\gamma \sigma}(t) r^{-1 / \gamma}(t) \omega^{(\gamma+1) / \gamma}(t) \leq 0, \quad t \in\left[t_{1}, \infty\right)_{\mathbb{T}} .
$$

Integrating it from $t_{1}$ to $t$, we get

$$
\int_{t_{1}}^{t} R^{\gamma \sigma}(s) \omega^{\Delta}(s) \Delta s+\int_{t_{1}}^{t} p(s)\left(\frac{m(g(s))}{m(\sigma(s))}\right)^{\gamma} R^{\gamma \sigma}(s) \Delta s+\gamma \int_{t_{1}}^{t} R^{\gamma \sigma}(s) r^{-1 / \gamma}(s) \omega^{(\gamma+1) / \gamma}(s) \Delta s \leq 0 .
$$

Integrating by parts, we have

$$
\int_{t_{1}}^{t} R^{r \sigma}(s) \omega^{\Delta}(s) \Delta s=R^{\gamma}(t) \omega(t)-R^{\gamma}\left(t_{1}\right) \omega\left(t_{1}\right)-\int_{t_{1}}^{t}\left(R^{\gamma}(s)\right)^{\Delta} \omega(s) \Delta s .
$$

From Keller's chain rule [3, Theorem 1.90], we obtain

$$
\left(R^{\gamma}(t)\right)^{\Delta}=\gamma \int_{0}^{1}\left[h R^{\sigma}(t)+(1-h) R(t)\right]^{\gamma-1} \mathrm{~d} h R^{\Delta}(t) .
$$

Note that $R^{\Delta}(t)=-(1 / r(t))^{1 / \gamma}<0$, we get

$$
-\int_{t_{1}}^{t}\left(R^{\gamma}(s)\right)^{\Delta} \omega(s) \Delta s \geq \gamma \int_{t_{1}}^{t}\left(\frac{1}{r(s)}\right)^{1 / \gamma}\left(R^{\sigma}(s)\right)^{\gamma-1} \omega(s) \Delta s
$$


due to (3.24) and $r \leq 1$. By (3.22), (3.23), and (3.25), we see that

$$
\begin{aligned}
& R^{\gamma}(t) \omega(t)-R^{\gamma}\left(t_{1}\right) \omega\left(t_{1}\right)+\int_{t_{1}}^{t} p(s)\left(\frac{m(g(s))}{m(\sigma(s))}\right)^{\gamma} R^{\gamma \sigma}(s) \Delta s+\gamma \int_{t_{1}}^{t}\left(\frac{1}{r(s)}\right)^{1 / \gamma}\left(R^{\sigma}(s)\right)^{\gamma-1} \omega(s) \Delta s \\
& \quad+\gamma \int_{t_{1}}^{t} R^{\gamma \sigma}(s) r^{-1 / \gamma}(s) \omega^{(\gamma+1) / \gamma}(s) \Delta s \leq 0 .
\end{aligned}
$$

Set $p:=(\gamma+1) / \gamma, q:=\gamma+1$,

$$
\begin{gathered}
A:=-(\gamma+1)^{\gamma /(\gamma+1)}\left(\frac{R^{\gamma \sigma}(t)}{r^{1 / \gamma}(t)}\right)^{\gamma /(\gamma+1)} \omega(t), \\
B:=\frac{\gamma}{\gamma+1}(\gamma+1)^{1 /(\gamma+1)}\left(\frac{1}{r^{1 / \gamma}(t)}\right)^{1 /(\gamma+1)} \frac{1}{\left(R^{\sigma}(t)\right)^{1 /(\gamma+1)}} .
\end{gathered}
$$

Then, using the inequality

$$
\frac{A^{p}}{p}+\frac{B^{q}}{q} \geq A B, \quad \frac{1}{p}+\frac{1}{q}=1
$$

we have

$$
\gamma R^{\gamma \sigma}(t) r^{-1 / \gamma}(t) \omega^{(\gamma+1) / \gamma}(t)+\left(\frac{\gamma}{\gamma+1}\right)^{\gamma+1} \frac{1}{R^{\sigma}(t) r^{1 / \gamma}(t)} \geq-\gamma\left(\frac{1}{r(t)}\right)^{1 / \gamma}\left(R^{\sigma}(t)\right)^{\gamma-1} \omega(t) .
$$

Thus, by (3.26) and (3.29), we get

$$
R^{\gamma}(t) \omega(t)-R^{\gamma}\left(t_{1}\right) \omega\left(t_{1}\right)+\int_{t_{1}}^{t}\left[p(s)\left(\frac{m(g(s))}{m(\sigma(s))}\right)^{\gamma} R^{\gamma \sigma}(s)-\left(\frac{\gamma}{\gamma+1}\right)^{\gamma+1} \frac{1}{R^{\sigma}(s) r^{1 / \gamma}(s)}\right] \Delta s \leq 0
$$

which contradicts (3.5) due to (3.12). The proof is complete.

$$
\text { If } g(t)=t \text {, similar as in the proof of Theorem 3.2, we obtain the following result. }
$$

Theorem 3.3. Assume that (1.10) holds, $g(t)=t$ and $\gamma \leq 1$. Furthermore, assume that there exists a positive real-valued $\Delta$-differentiable function $\delta$ such that (3.4) holds for all sufficiently large $T$. If

$$
\limsup _{t \rightarrow \infty} \int_{T}^{t}\left[p(s) R^{\gamma \sigma}(s)-\left(\frac{\gamma}{\gamma+1}\right)^{\gamma+1} \frac{1}{R^{\sigma}(s) r^{1 / \gamma}(s)}\right] \Delta s=\infty
$$

then (1.1) is oscillatory. 
Theorem 3.4. Assume that (1.10) holds, $g(t) \geq \sigma(t)$ and $r \geq 1$. Suppose further that there exists a positive real-valued $\Delta$-differentiable function $\delta$ such that (3.4) holds for all sufficiently large $T$. If

$$
\limsup _{t \rightarrow \infty} \int_{T}^{t}\left[p(s)\left(\frac{m(g(s))}{m(\sigma(s))}\right)^{\gamma} R^{\gamma \sigma}(s)-\left(\frac{r}{\gamma+1}\right)^{\gamma+1} \frac{R^{r^{2}-1}(s)}{\left(R^{\sigma}(s)\right)^{\gamma^{2}} r^{1 / \gamma}(s)}\right] \Delta s=\infty,
$$

then (1.1) is oscillatory.

Proof. Let $x$ be a nonoscillatory solution of (1.1). Without loss of generality, we may assume that $x(t)>0$ and $x(g(t))>0$ for $t \in\left[t_{0}, \infty\right)_{\mathbb{T}}$. In view of (1.1), we obtain (3.6). Therefore, $r(t)\left(x^{\Delta}(t)\right)^{\gamma}$ is an eventually strictly decreasing function, and so there exists a $t_{1} \in\left[t_{0}, \infty\right)_{\mathbb{T}}$ such that $x^{\Delta}(t)>0, t \in\left[t_{1}, \infty\right)_{\mathbb{T}}$ or $x^{\Delta}(t)<0, t \in\left[t_{1}, \infty\right)_{\mathbb{T}}$.

Case 1. Assume that $x^{\Delta}(t)>0, t \in\left[t_{1}, \infty\right)_{\mathbb{T}}$. From Theorem A, we can obtain a contradiction to (3.4).

Case 2. Assume that $x^{\Delta}(t)<0, t \in\left[t_{1}, \infty\right)_{\mathbb{T}}$. Define $\omega$ as in (3.7). We have (3.12). $\Delta$ differentiating (3.7) and using (3.6), we obtain (3.16). In view of Keller's chain rule [3, Theorem 1.90], we see that

$$
\left(x^{\gamma}(t)\right)^{\Delta} \leq \gamma\left(x^{\sigma}(t)\right)^{\gamma-1} x^{\Delta}(t), \quad \text { since } \gamma \geq 1 \text {. }
$$

Thus, we get

$$
\omega^{\Delta}(t) \leq-p(t)\left(\frac{m(g(t))}{m(\sigma(t))}\right)^{\gamma}-\gamma \frac{r(t)\left(x^{\Delta}(t)\right)^{\gamma+1}}{x^{\gamma}(t) x(\sigma(t))} .
$$

On the other hand, from $x^{\Delta}(t)<0$, we have $x(t) \geq x^{\sigma}(t)$ and

$$
-\gamma \frac{r(t)\left(x^{\Delta}(t)\right)^{\gamma+1}}{x^{\gamma}(t) x(\sigma(t))} \leq-\gamma\left(\frac{1}{r(t)}\right)^{1 / \gamma} \omega^{(\gamma+1) / \gamma}(t) .
$$

Hence by (3.34), we get (3.20). Then we obtain that (3.22) and (3.23) hold. By Keller's chain rule [3, Theorem 1.90], we have (3.24). From (3.24), $\gamma \geq 1$ and $R^{\Delta}(t)=-(1 / r(t))^{1 / \gamma}<0$, we see that

$$
-\int_{t_{1}}^{t}\left(R^{\gamma}(s)\right)^{\Delta} \omega(s) \Delta s \geq \gamma \int_{t_{1}}^{t}\left(\frac{1}{r(s)}\right)^{1 / \gamma} R^{\gamma-1}(s) \omega(s) \Delta s .
$$


It follows from (3.22), (3.23), and (3.36) that

$$
\begin{aligned}
& R^{\gamma}(t) \omega(t)-R^{\gamma}\left(t_{1}\right) \omega\left(t_{1}\right)+\int_{t_{1}}^{t} p(s)\left(\frac{m(g(s))}{m(\sigma(s))}\right)^{\gamma} R^{\gamma \sigma}(s) \Delta s+\gamma \int_{t_{1}}^{t}\left(\frac{1}{r(s)}\right)^{1 / \gamma} R^{\gamma-1}(s) \omega(s) \Delta s \\
& \quad+\gamma \int_{t_{1}}^{t} R^{\gamma \sigma}(s) r^{-1 / \gamma}(s) \omega^{(\gamma+1) / \gamma}(s) \Delta s \leq 0 .
\end{aligned}
$$

Set $p:=(\gamma+1) / \gamma, q:=\gamma+1$,

$$
\begin{gathered}
A:=-(\gamma+1)^{\gamma /(\gamma+1)}\left(\frac{R^{\gamma \sigma}(t)}{r^{1 / \gamma}(t)}\right)^{\gamma /(\gamma+1)} \omega(t), \\
B:=\frac{\gamma}{\gamma+1}(\gamma+1)^{1 /(\gamma+1)}\left(\frac{1}{r^{1 / \gamma}(t)}\right)^{1 /(\gamma+1)} \frac{R^{\gamma-1}(t)}{\left(R^{\sigma}(t)\right)^{\gamma^{2} /(\gamma+1)}} .
\end{gathered}
$$

By the inequality (3.28), we have

$$
\gamma R^{\gamma \sigma}(t) r^{-1 / \gamma}(t) \omega^{(\gamma+1) / \gamma}(t)+\left(\frac{\gamma}{\gamma+1}\right)^{\gamma+1} \frac{R^{r^{2}-1}(t)}{\left(R^{\sigma}(t)\right)^{\gamma^{2}} r^{1 / \gamma}(t)} \geq-\gamma\left(\frac{1}{r(t)}\right)^{1 / \gamma} R^{\gamma-1}(t) \omega(t) .
$$

Thus, (3.37) and (3.39) implies

$$
R^{\gamma}(t) \omega(t)-R^{\gamma}\left(t_{1}\right) \omega\left(t_{1}\right)+\int_{t_{1}}^{t}\left[p(s)\left(\frac{m(g(s))}{m(\sigma(s))}\right)^{\gamma} R^{\gamma \sigma}(s)-\left(\frac{r}{\gamma+1}\right)^{\gamma+1} \frac{R^{r^{2}-1}(s)}{\left(R^{\sigma}(s)\right)^{\gamma^{2}} r^{1 / \gamma}(s)}\right] \Delta s \leq 0 .
$$

which contradicts (3.32) when using (3.12). This completes the proof.

If $g(t)=t$, similar as in the proof of Theorem 3.4, we establish the following result.

Theorem 3.5. Assume that (1.10) holds, $g(t)=t$ and $\gamma \geq 1$. Moreover, assume that there exists a positive real-valued $\Delta$-differentiable function $\delta$ such that (3.4) holds for all sufficiently large $T$. If

$$
\limsup _{t \rightarrow \infty} \int_{T}^{t}\left[p(s) R^{\gamma \sigma}(s)-\left(\frac{\gamma}{\gamma+1}\right)^{\gamma+1} \frac{R^{\gamma^{2}-1}(s)}{\left(R^{\sigma}(s)\right)^{\gamma^{2}} r^{1 / \gamma}(s)}\right] \Delta s=\infty
$$

then (1.1) is oscillatory. 
Theorem 3.6. Assume that (1.10) holds, $g(t) \geq \sigma(t)$ and $\gamma>0$. Assume further that there exists a positive real-valued $\Delta$-differentiable function $\delta$ such that (3.4) holds for all sufficiently large $T$. If

$$
\int_{T}^{t} p(s)\left(\frac{m(g(s))}{m(\sigma(s))}\right)^{\gamma} R^{\gamma+1}(\sigma(s)) \Delta s=\infty
$$

then (1.1) is oscillatory.

Proof. Let $x$ be a nonoscillatory solution of (1.1). Without loss of generality, we assume that $x(t)>0$ and $x(g(t))>0$ for $t \in\left[t_{0}, \infty\right)_{\mathbb{T}}$. Similar as in the proof of Theorem 3.2 or Theorem 3.4, we consider two cases.

Case 1. Assume $x^{\Delta}(t)>0, t \in\left[t_{1}, \infty\right)_{\mathbb{T}}$. By (3.4), this case is not true.

Case 2. Suppose $x^{\Delta}(t)<0, t \in\left[t_{1}, \infty\right)_{\mathbb{T}}$. If $\gamma \leq 1$, proceeding as in the proof of Case 2 of Theorem 3.2, we obtain (3.12) and (3.20). Multiplying (3.20) by $R^{r+1}(\sigma(t))$, and integrating it from $t_{1}$ to $t$, we get

$$
\begin{aligned}
& \int_{t_{1}}^{t} R^{\gamma+1}(\sigma(s)) \omega^{\Delta}(s) \Delta s+\int_{t_{1}}^{t} p(s)\left(\frac{m(g(s))}{m(\sigma(s))}\right)^{\gamma} R^{\gamma+1}(\sigma(s)) \Delta s \\
& \quad+\gamma \int_{t_{1}}^{t} R^{\gamma+1}(\sigma(s)) r^{-1 / \gamma}(s) \omega^{(\gamma+1) / \gamma}(s) \Delta s \leq 0 .
\end{aligned}
$$

Integrating by parts, we see that

$$
\int_{t_{1}}^{t} R^{\gamma+1}(\sigma(s)) \omega^{\Delta}(s) \Delta s=R^{\gamma+1}(t) \omega(t)-R^{\gamma+1}\left(t_{1}\right) \omega\left(t_{1}\right)-\int_{t_{1}}^{t}\left(R^{\gamma+1}(s)\right)^{\Delta} \omega(s) \Delta s .
$$

By Keller's chain rule [3, Theorem 1.90], we obtain

$$
\left(R^{\gamma+1}(t)\right)^{\Delta}=(\gamma+1) \int_{0}^{1}\left[h R^{\sigma}(t)+(1-h) R(t)\right]^{\gamma} \mathrm{d} h R^{\Delta}(t) .
$$

Note that $R^{\Delta}(t)=-(1 / r(t))^{1 / \gamma}<0$, we have

$$
-\int_{t_{1}}^{t}\left(R^{r+1}(s)\right)^{\Delta} \omega(s) \Delta s \geq(\gamma+1) \int_{t_{1}}^{t}\left(\frac{1}{r(s)}\right)^{1 / \gamma} R^{r}(s) \omega(s) \Delta s .
$$


Thus, from (3.44), we get

$$
\begin{aligned}
& R^{\gamma+1}(t) \omega(t)-R^{\gamma+1}\left(t_{1}\right) \omega\left(t_{1}\right)+\int_{t_{1}}^{t} p(s)\left(\frac{m(g(s))}{m(\sigma(s))}\right)^{\gamma} R^{\gamma+1}(\sigma(s)) \Delta s \\
& \quad+(\gamma+1) \int_{t_{1}}^{t}\left(\frac{1}{r(s)}\right)^{1 / \gamma} R^{\gamma}(s) \omega(s) \Delta s+\gamma \int_{t_{1}}^{t} R^{\gamma+1}(\sigma(s)) r^{-1 / \gamma}(s) \omega^{(\gamma+1) / \gamma}(s) \Delta s \leq 0 .
\end{aligned}
$$

It follows from (3.12) that

$$
\begin{gathered}
-R^{r+1}(t) \omega(t) \leq R(t)<\infty, \quad t \longrightarrow \infty \\
-\int_{t_{1}}^{\infty}\left(\frac{1}{r(s)}\right)^{1 / \gamma} R^{r}(s) \omega(s) \Delta s \leq \int_{t_{1}}^{\infty}\left(\frac{1}{r(s)}\right)^{1 / \gamma} \Delta s<\infty .
\end{gathered}
$$

Noting that $R^{\sigma}(t) / R(t) \leq 1$, we obtain

$$
\begin{aligned}
\int_{t_{1}}^{\infty} R^{\gamma+1}(\sigma(s)) r^{-1 / \gamma}(s) \omega^{(\gamma+1) / \gamma}(s) \Delta s & =\int_{t_{1}}^{\infty} r^{-1 / \gamma}(s)\left(\frac{R^{\sigma}(s)}{R(s)}\right)^{\gamma+1}\left(R^{\gamma}(s) \omega(s)\right)^{(\gamma+1) / \gamma} \Delta s \\
& \leq \int_{t_{1}}^{\infty} r^{-1 / \gamma}(s) \Delta s<\infty .
\end{aligned}
$$

Thus, from (3.47), we get

$$
\int_{t_{1}}^{\infty} p(s)\left(\frac{m(g(s))}{m(\sigma(s))}\right)^{\gamma} R^{\gamma+1}(\sigma(s)) \Delta s<\infty,
$$

which contradicts (3.42).

When $\gamma \geq 1$, the proof is similar to that of the case where $\gamma \leq 1$, so we omit the details. The proof is complete.

If $g(t)=t$, similar as in the proof of Theorem 3.6, we have the following result.

Theorem 3.7. Assume that (1.10) holds, $g(t)=t$ and $\gamma>0$. Furthermore, assume that there exists a positive real-valued $\Delta$-differentiable function $\delta$ such that (3.4) holds for all sufficiently large $T$. If

$$
\int_{T}^{\infty} p(s) R^{r+1}(\sigma(s)) \Delta s=\infty,
$$

then (1.1) is oscillatory.

\section{Examples}

For some applications, we give the following examples. 
Example 4.1. Consider the second-order linear dynamic equation

$$
\left(t \sigma(t) x^{\Delta}(t)\right)^{\Delta}+\lambda \frac{\sigma(t)}{t} x(\sigma(t))=0, \quad t \in[1, \infty)_{\mathbb{T}}
$$

where $\lambda>0$ is a constant.

Let

$$
r(t)=t \sigma(t), \quad p(t)=\lambda \frac{\sigma(t)}{t}, \quad g(t)=\sigma(t) .
$$

Then $R(t)=1 / t$. Set $m(t)=R(t)=1 / t$ and $\delta(t)=1$. Using Theorem 3.2, it is easy to see that every solution of (4.1) is oscillatory if $\lambda>1 / 4$. This result extends that of [20]. But recent results on the oscillation of second-order dynamic equations on time scales cannot be applied in (4.1).

Example 4.2. Consider the second-order advanced differential equation

$$
\left(t^{2} x^{\prime}(t)\right)^{\prime}+p_{0} x(2 t)=0, \quad t \geq 1
$$

where $p_{0}>0$ is a constant.

Let $m(t)=t^{-1}$. Applying Theorem 3.2, we see that (4.3) is oscillatory if $p_{0}>1 / 2$. However, results of [15] cannot be applied to (4.3), since

$$
\int_{1}^{\infty}\left[\frac{1}{r(t)} \int_{1}^{t} p(s)\left(\int_{g(s)}^{\infty} \frac{\mathrm{d} u}{r^{1 / \gamma}(u)}\right)^{\gamma} \mathrm{d} s\right]^{1 / \gamma} \mathrm{d} t<\infty .
$$

Example 4.3. Consider the second-order superlinear advanced dynamic equation

$$
\left((t \sigma(t))^{\gamma}\left(x^{\Delta}(t)\right)^{\gamma}\right)^{\Delta}+\lambda \frac{g^{\gamma}(t)}{t} x^{\gamma}(g(t))=0, \quad t \in[1, \infty)_{\mathbb{T}},
$$

where $\gamma \geq 1$ is the ratio of odd positive integers, $\lambda>0$ is a constant, $k_{1} t \leq \sigma(t) \leq k_{2} t$, and $g(t) \geq \sigma(t)$.

Let

$$
r(t)=(t \sigma(t))^{\gamma}, \quad p(t)=\lambda \frac{g^{\gamma}(t)}{t}
$$


Then $R(t)=1 / t$. Set $\delta(t)=1$. Clearly, (3.4) holds. Put $m(t)=R(t)=1 / t$. Then

$$
\begin{aligned}
& \limsup _{t \rightarrow \infty} \int_{T}^{t}\left[p(s)\left(\frac{m(g(s))}{m(\sigma(s))}\right)^{\gamma} R^{\gamma \sigma}(s)-\left(\frac{\gamma}{\gamma+1}\right)^{\gamma+1} \frac{R^{\gamma^{2}-1}(s)}{\left(R^{\sigma}(s)\right)^{\gamma^{2}} r^{1 / \gamma}(s)}\right] \Delta s \\
& \quad=\limsup _{t \rightarrow \infty} \int_{T}^{t}\left[\lambda \frac{g^{\gamma}(s)}{s} \frac{\sigma^{\gamma}(s)}{g^{\gamma}(s)} \frac{1}{\sigma^{\gamma}(s)}-\left(\frac{\gamma}{\gamma+1}\right)^{\gamma+1}\left(\frac{\sigma(s)}{s}\right)^{\gamma^{2}} \frac{1}{\sigma(s)}\right] \Delta s \\
& \quad \geq\left(\lambda-\left(\frac{\gamma}{\gamma+1}\right)^{\gamma+1} \frac{k_{2}^{\gamma^{2}}}{k_{1}}\right) \limsup _{t \rightarrow \infty} \int_{T}^{t} \frac{1}{s} \Delta s \\
& \quad=\infty
\end{aligned}
$$

if

$$
\lambda>\left(\frac{r}{r+1}\right)^{\gamma+1} \frac{k_{2} r^{2}}{k_{1}}
$$

Therefore, by Theorem 3.4, every solution of (4.5) is oscillatory when the above inequality holds.

\section{Summary}

This paper is concerned with the oscillatory behavior of advanced equation (1.1). By using the generalized Riccati substitution, we establish some new oscillation criteria for (1.1). On one hand, these criteria can be extended to the equation of the form

$$
\left(r(t)\left(x^{\Delta}(t)\right)^{\gamma}\right)^{\Delta}+p(t) x^{\beta}(g(t))=0 .
$$

On the other hand, the main results supplement and improve those results of [9] and extend those results of [20]. The established results are easily applicable and are illustrated on three suitable examples.

\section{Acknowledgments}

The authors sincerely thank the reviewers and Professor Dumitru Baleanu for their valuable suggestions and useful comments that have led to the present improved version of the original manuscript.

\section{References}

[1] S. Hilger, "Analysis on measure chains—a unified approach to continuous and discrete calculus," Results in Mathematics, vol. 18, no. 1-2, pp. 18-56, 1990. 
[2] R. Agarwal, M. Bohner, D. O'Regan, and A. Peterson, "Dynamic equations on time scales: a survey," Journal of Computational and Applied Mathematics, vol. 141, no. 1-2, pp. 1-26, 2002.

[3] M. Bohner and A. Peterson, Dynamic Equations on Time Scales, Birkhäuser, Boston, Mass, USA, 2001.

[4] M. Bohner and A. Peterson, Advances in Dynamic Equations on Time Scales, Birkhäuser, Boston, Mass, USA, 2003.

[5] R. P. Agarwal, D. O'Regan, and S. H. Saker, "Philos-type oscillation criteria for second order halflinear dynamic equations on time scales," The Rocky Mountain Journal of Mathematics, vol. 37, no. 4, pp. 1085-1104, 2007.

[6] S. R. Grace, M. Bohner, and R. P. Agarwal, "On the oscillation of second-order half-linear dynamic equations," Journal of Difference Equations and Applications, vol. 15, no. 5, pp. 451-460, 2009.

[7] S. R. Grace, R. P. Agarwal, B. Kaymakçalan, and W. Sae-jie, “On the oscillation of certain second order nonlinear dynamic equations," Mathematical and Computer Modelling, vol. 50, no. 1-2, pp. 273-286, 2009.

[8] S. H. Saker, "Oscillation criteria of second-order half-linear dynamic equations on time scales," Journal of Computational and Applied Mathematics, vol. 177, no. 2, pp. 375-387, 2005.

[9] T. S. Hassan, "Oscillation criteria for half-linear dynamic equations on time scales," Journal of Mathematical Analysis and Applications, vol. 345, no. 1, pp. 176-185, 2008.

[10] Y. Şahiner, "Oscillation of second-order delay differential equations on time scales," Nonlinear Analysis: Theory, Methods and Applications, vol. 63, no. 5-7, pp. e1073-e1080, 2005.

[11] L. Erbe, A. Peterson, and S. H. Saker, "Oscillation criteria for second-order nonlinear delay dynamic equations," Journal of Mathematical Analysis and Applications, vol. 333, no. 1, pp. 505-522, 2007.

[12] L. Erbe, T. S. Hassan, and A. Peterson, "Oscillation criteria for nonlinear functional neutral dynamic equations on time scales," Journal of Difference Equations and Applications, vol. 15, no. 11-12, pp. 10971116, 2009.

[13] T. Li, Z. Han, S. Sun, and C. Zhang, "Forced oscillation of second-order nonlinear dynamic equations on time scales," Electronic Journal of Qualitative Theory of Differential Equations, vol. 60, pp. 1-8, 2009.

[14] S. H. Saker, "Oscillation of nonlinear dynamic equations on time scales," Applied Mathematics and Computation, vol. 148, no. 1, pp. 81-91, 2004.

[15] T. S. Hassan, "Kamenev-type oscillation criteria for second order nonlinear dynamic equations on time scales," Applied Mathematics and Computation, vol. 217, no. 12, pp. 5285-5297, 2011.

[16] E. Thandapani and K. Ravi, "Oscillation of second-order half-linear difference equations," Applied Mathematics Letters, vol. 13, no. 2, pp. 43-49, 2000.

[17] E. Thandapani, K. Ravi, and J. R. Graef, "Oscillation and comparison theorems for half-linear secondorder difference equations," Computers $\mathcal{E}$ Mathematics with Applications, vol. 42, no. 6-7, pp. 953-960, 2001.

[18] H. A. Agwo, A. M. M. Khodier, and H. A. Hassan, “Oscillation of second-order nonlinear delay dynamic equations on time scales," International Journal of Differential Equations. In press.

[19] D. Willett, "On the oscillatory behavior of the solutions of second order linear differential equations," Polska Akademia Nauk, vol. 21, pp. 175-194, 1969.

[20] H. J. Li, "Oscillation criteria for second order linear differential equations," Journal of Mathematical Analysis and Applications, vol. 194, no. 1, pp. 217-234, 1995.

[21] M. Bohner and S. H. Saker, "Oscillation of damped second order nonlinear delay differential equations of Emden-Fowler type," Advances in Dynamical Systems and Applications, vol. 1, no. 2, pp. 163-182, 2006.

[22] M. Budinčević, "Oscillations and the asymptotic behaviour of certain second order neutral difference equations," Zbornik Radova Prirodno-Matematichkog Fakulteta, vol. 21, no. 1, pp. 165-172, 1991. 


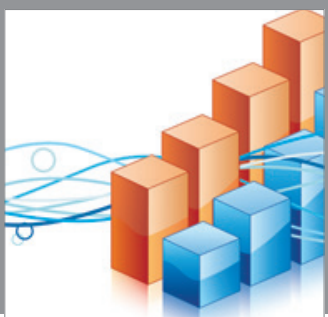

Advances in

Operations Research

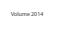

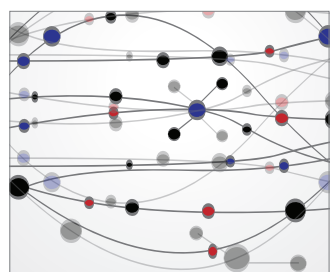

\section{The Scientific} World Journal
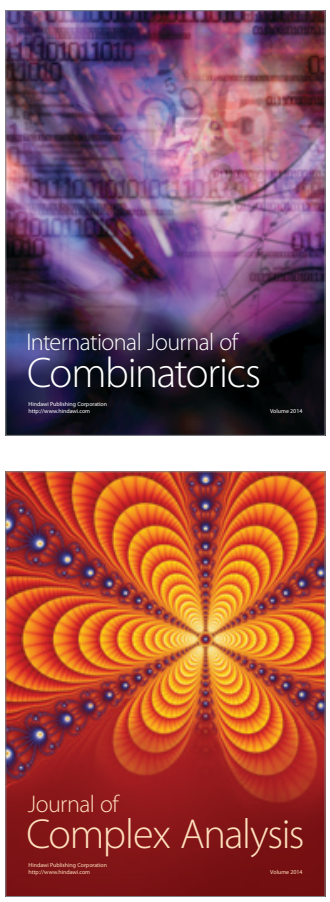

International Journal of

Mathematics and

Mathematical

Sciences
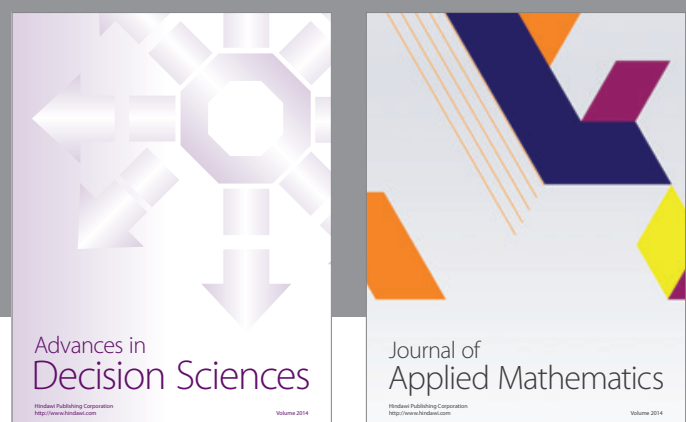

Journal of

Applied Mathematics
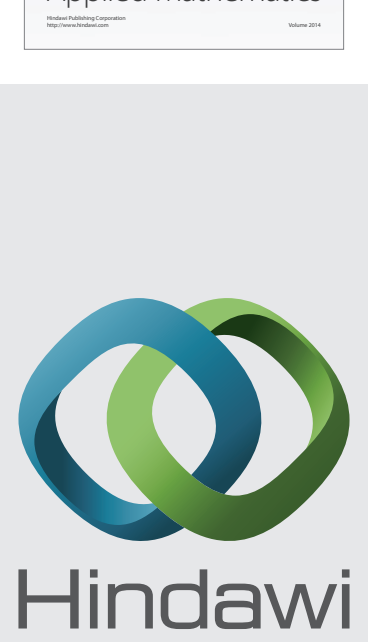

Submit your manuscripts at http://www.hindawi.com
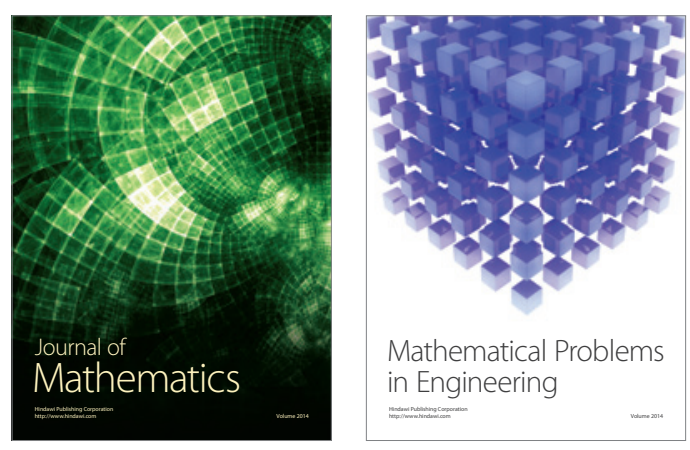

Mathematical Problems in Engineering
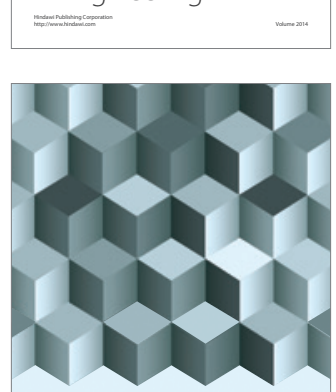

Journal of

Function Spaces
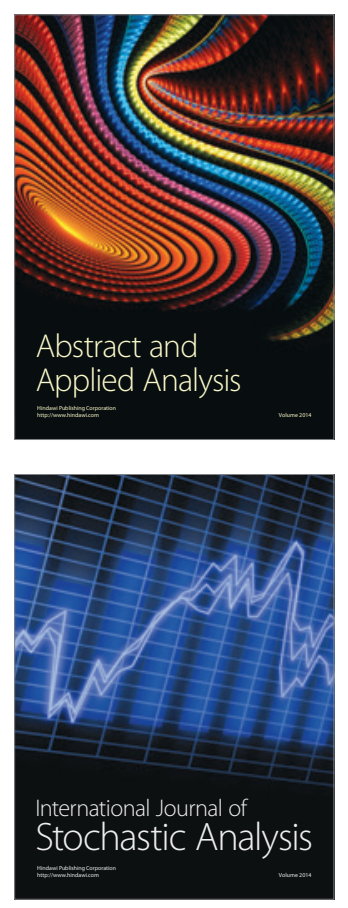

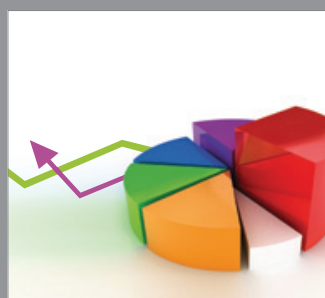

ournal of

Probability and Statistics

Promensencen
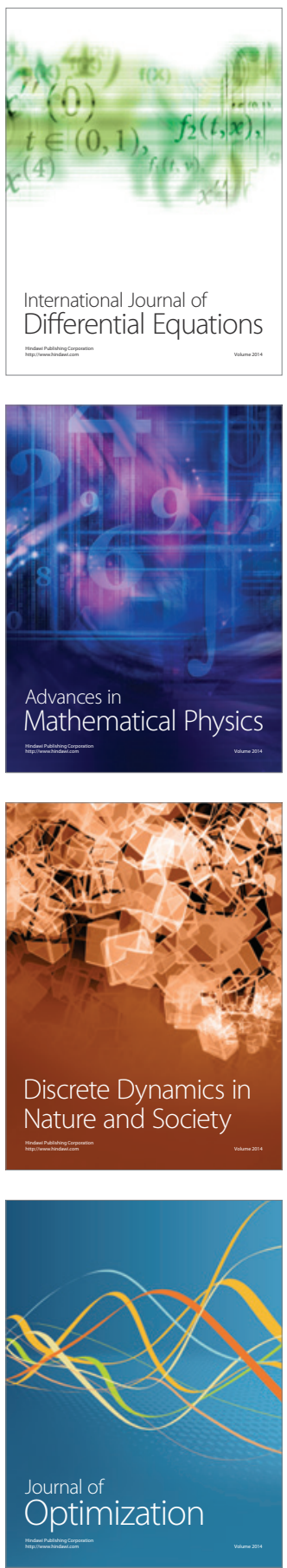